\title{
Copulas, Chaotic Processes and Time Series: a Survey
}

\section{Guilherme Pumi}

Mathematics Institute - Federal University of Rio Grande do Sul - 9500, Bento Gonçalves Avenue - 91509-900 Porto Alegre - RS - Brazil.

E-mail address: guipumi@gmail.com

\section{Sílvia R.C. Lopes}

Mathematics Institute - Federal University of Rio Grande do Sul - 9500, Bento Gonçalves Avenue - 91509-900 Porto Alegre - RS - Brazil.

E-mail address: silvia.lopes@ufrgs.br

\begin{abstract}
In this work we summarize some of recent and classical results on the role played by copulas in the analysis of chaotic processes and univariate time series. We review some aspects of the copulas related to chaotic process, its properties and applications. We also present a review on classical and modern approaches to understand the relationship among random variables in Markov processes as well as short and long memory time series as well as ergodic properties of copula-based Markov processes.
\end{abstract}

Keywords. Chaotic Processes, Copulas, Interval Maps, Invariant Measures, Parametric Estimation.

\section{Introduction}

This paper aims to present a brief survey on the study of copulas and its relationship with chaotic process and univariate time series. This work does not intend to be complete or in-depth, but rather to reflect the preferences and the research conducted by the authors on these subjects.

For any set of random variables $X_{1}, \cdots, X_{n}$ with joint distribution function $H$ and marginals $F_{1}, \cdots, F_{n}$, respectively, copulas are a very helpful tool for understanding statistical properties of these family of distributions and, consequently, of the vector $\left(X_{1}, \cdots, X_{n}\right)^{\prime}$. 
The name "copula" dates back to the work of Sklar (1959), where the celebrated Sklar's theorem was first proven. The use of such functions, however, predates Sklar's work. Sklar's theorem is considered the most important theorem in the theory because it elucidates the role played by copulas on "coupling", that is, tieing together any multivariate distribution with its marginals. Sklar's theorem is also the base of most, if not all, applications of copulas in statistics and stochastic processes. The reader interested on the history of copulas is referred to Schweizer (1991), Schweizer and Sklar (2005), Nelsen (2006) and references therein.

The interest on copulas in late 1950's to early 1980's was motivated by the development of the theory of probabilistic metric spaces. Although most of the crucial theorems on the theory of copulas dates back to that epoch, the theory of copulas became dormant for almost a decade when in late 1980's to early 1990's, the discovery of new applications of copulas in finances and statistics, renewed the interest on them. After late 1990's the theory and application of copulas and its ramifications to stochastic processes grew enormously. For an account of these developments see Nelsen (2006) and Cherubini et al. (2004). More details on copulas will be provided in Section 2 .

Consider a smooth function $T: I \rightarrow I$, where $I:=[0,1]$, and assume $\mu_{T}$ is a $T$-invariant probability measure. Let $U_{0}$ be a random variable distributed as $\mu_{T}$. We can define a stochastic process by setting

$$
X_{t}:=\varphi\left(T^{t}\left(U_{0}\right)\right), t \in \mathbb{N},
$$

for a given $\mu_{T}$-integrable function $\varphi:[0,1] \rightarrow \mathbb{R}$. This is often called a (pure) chaotic process. In the literature, however, sometimes a signal plus noise type of process where the signal is the process defined in (1.1) plus a random noise is considered. In this work we shall call a chaotic process such the one defined in (1.1).

From the definition of chaotic process, we observe that, for a given fixed $\omega, X_{t}(\omega)=\varphi\left(T^{t}\left(U_{0}(\omega)\right)\right)$ and, from setting $w_{0}:=U_{0}(\omega)$, we see that the trajectory $\left\{X_{t}(\omega)\right\}_{t \in \mathbb{N}}$ is the avaliation of $\varphi$ in the $t$-th iteration of $T$ in the initial point $w_{0}$. This means that a realization of $\left\{X_{t}\right\}_{t \in \mathbb{N}}$ can be seen as a deterministically generated sequence. Nevertheless, a realization of such process present a complex/chaotic dynamics, with very unstable and erratic behavior and instability with respect to the initial point $w_{0}=U_{0}(\omega)$. This type of process has been applied on a variety of problems from rock drilling to the study of intermittency on human cardiac rate (see Zebrowsky, 2001, Lasota and Mackey, 1994 and references therein). As an illustration, Figure 1 shows two sample paths of the Manneville-Pomeau process for two initial points distant 0.0001 of one another. Notice that after 10 steps or so the two sample paths become far apart. 


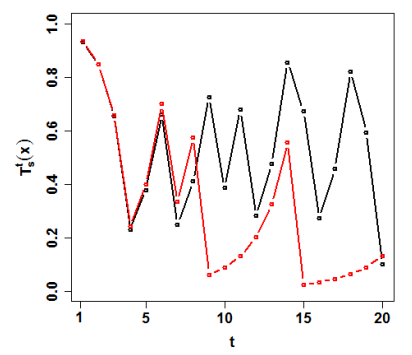

Figure 1. Two realizations of the Manneville-Pomeau process for 2 distinct initial points distant $10^{-3}$ from each other.

Although the literature on dynamical system and ergodic theory related to these type of processes is vast (see Lasota and Mackey, 1994, Pollicott and Yuri, 1998 and references therein), the statistics aspects related to such processes is still in its infancy and has seen grown attention in the last decade (see, for instance, Chazottes et al., 2005 and references therein). Olbermann et al. (2007), Lopes and Pumi (2013) and Pumi and Lopes (2013a) discuss parameter estimation on chaotic process while parameter estimation on a signal plus noise setting is discussed in Lopes and Lopes (1998).

The connection between copulas and dynamical systems/ergodic theory is also a subject not very much explored. Some works on this direction are Kolesárová et al. (2008), Lopes and Pumi (2013), Pumi and Lopes (2013a) and Schmidl et al. (2013).

The interest in the connections between copulas and stochastic process/time series has also seem a grown of attention in the last years. Most of the literature is based on the seminal paper on copula-based Markov processes by Darsow et al. (1992) and later development by Ibragimov (2009) on copula-based higher order Markov processes. See also Pumi and Lopes (2013b) and references therein.

Our paper is organized as follows: in the next section we consider some preliminaries results necessary to the work. Section 3 presents some nomenclature and review some facts on chaotic process while in Section 4 we discuss the copulas related to certain chaotic processes, approximations and applications in parameter estimation in these processes. In Section 5 we review the literature on copula-based Markov process as well as consider some extensions to time series with non-Markovian dependence, including long-range dependent ones. In Section 6 we present an application and Section 7 concludes the paper. 


\section{Preliminaries}

We start by recalling some facts on copulas. An $n$-dimensional copula is a distribution function for which the marginals are uniformly distributed on $I$ and whose support is contained in the hypercube $I^{n}$. We recall that the support is the whole hypercube $I^{n}$ only when the independence copula is considered. The usefulness of copulas in applications lies on its capability to allow the manipulation of the joint and marginal dependence structure separately from each other, leading to great flexibility.

Copulas have been successfully applied and widely spread in several areas. In finances, copulas have been applied, and become standard tools, in major topics such as asset pricing, risk management and credit risk analysis among many others (see the books by Cherubini et al., 2004 and McNeil et al., 2005 for details). In hydrology, the modeling of rainfalls and storms often employ copulas to describe joint features among variables in the models (see, for instance, Palynchuk and Guo, 2011 and references therein). In econometrics, copulas have been widely employed in constructing multidimensional extensions of complex models (see, for instance Lee and Long, 2009, Wang et al., 2009 and references therein). In statistics, copulas have been applied in all sort of problems, such as development of dependence measures, modeling, testing, just to cite a few (see Mari and Kotz, 2001, Nelsen, 2006 and references therein). It has also been successfully applied as a tool in simulation of time series and stochastic processes for which it is desired to compare different marginal structure under exactly the same joint structure and vice-versa. See, for instance, Lopes et al. (2013).

The main result in the theory of copulas is the celebrated Sklar's theorem, which we recall below. Sklar's theorem unravel the role played by copulas on coupling a multivariate distribution to its marginals and vice-versa. The original proof is due to Sklar (1959), but see Rüschendorf (2009) for a considerably simpler proof in the $n$-dimensional case and Nelsen (2006) for a more detailed proof in the bidimensional case on the lines of Sklar (1959).

Theorem 2.1 (Sklar's Theorem). Let $X_{1}, \cdots, X_{n}$ be random variables with joint distribution function $H$ and marginals $F_{1}, \cdots, F_{n}$, respectively. Then, there exists a copula $C$ such that

$$
H\left(x_{1}, \cdots, x_{n}\right)=C\left(F_{1}\left(x_{1}\right), \cdots, F_{n}\left(x_{n}\right)\right), \quad \text { for all }\left(x_{1}, \cdots, x_{n}\right) \in \mathbb{R}^{n} .
$$

If the $F_{i}$ 's are continuous, then $C$ is unique. Otherwise, $C$ is uniquely determined on $\operatorname{Ran}\left(F_{1}\right) \times \cdots \times \operatorname{Ran}\left(F_{n}\right)$, where $\operatorname{Ran}(F)$ denotes the range of the function $F$. The converse also holds. Furthermore,

$$
C\left(u_{1}, \cdots, u_{n}\right)=H\left(F_{1}^{(-1)}\left(u_{1}\right), \cdots, F_{n}^{(-1)}\left(u_{n}\right)\right), \quad \text { for all }\left(u_{1}, \cdots, u_{n}\right) \in I^{n},
$$

where for a function $F, F^{(-1)}$ denotes its pseudo-inverse given by $F^{(-1)}(x)$ $:=\inf \{u \in \operatorname{Ran}(F): F(u) \geq x\}$. 
For more details on the theory of copulas and its applications we refer to the books by Joe (1997), Cherubini et a. (2004), McNeil et al. (2005) and Nelsen (2006).

\section{Chaotic Processes}

Before proceeding further we need to establish some notation and some nomenclature, on the lines of Pumi and Lopes (2013a). We consider piecewise monotone maps with finitely many branches $T: I \rightarrow I$ for which each branch is of class $C^{1+\alpha}$, for $\alpha \in(0,1)$ (the class of differentiable functions whose derivatives are $\alpha$-Hölder continuous). Each branch is defined in $I_{k}$ which is assumed to be of the form $I_{k}:=\left[a_{k-1}, a_{k}\right), k=1, \cdots, s$ where $\left\{a_{0}, \cdots, a_{s}\right\}$ are the discontinuity points of $T$. We assume $T\left(I_{k}\right)=$ $T\left(\left[a_{k-1}, a_{k}\right)\right)=[0,1)$ is a bijection for each $k=1, \cdots, s$. Also, without loss of generality, $T$ will be assumed to be right continuous, except at 1, where it will be taken as left continuous. A map $T$ satisfying these conditions is called finitely piecewise monotone $C^{1+\alpha}$ function. We shall denote the set of finitely piecewise monotone $C^{1+\alpha}$ functions for which there exists an absolutely continuous invariant probability measure $\mu_{T}$ by $\mathcal{T}$.

Examples of piecewise monotone maps of such functions $T$ are the following:

(1) The Tent transformation with parameter $a \in(0,1)$ given by

$$
T_{a}(x):=\left\{\begin{array}{ccc}
\frac{x}{a}, & \text { if } & 0 \leq x<a, \\
\frac{1-x}{1-a}, & \text { if } & a \leq x \leq 1 .
\end{array}\right.
$$

It can be shown that the Lebesgue measure in $I$ is invariant. Figure 2(a) presents a typical graph of a Tent transformation. Lopes and Lopes (1995) consider large deviation properties, the spectral analysis and parameters $a$ and $\sigma_{\xi}$ estimation for the signal-plus-noise process given by $Z_{t}=X_{t}+\xi_{t}=\varphi\left(T_{a}\left(X_{t-1}\right)\right)+\xi_{t}$, when $\varphi(x)=x$ and the noise $\left\{\xi_{t}\right\}_{t \in \mathbb{N}}$ is Gaussian, for different signal-to-noise ratio values.

(2) The Giampieri-Isola transformation with parameter $r \in(0,1)$ given by

$$
T_{r}(x):=\left\{\begin{array}{clc}
\frac{(2-r) x}{1-r x}, & \text { if } & 0 \leq x \leq \frac{1}{2} \\
\frac{(2-r)(1-x)}{1-r(1-x)}, & \text { if } & \frac{1}{2}<x \leq 1 .
\end{array}\right.
$$

It can be shown that $f_{r}(x):=-\frac{r}{\log (1-r)(1-r+r x)}$ is the density of the absolute continuous and $T_{r}$-invariant probability measure $\mu_{T}$ 
(cf. Giampieri and Isola, 2005). Figure 2(b) presents a typical graph of a Giampieri-Isola transformation.

Recall that, the $T$-invariant probability measure considered here is absolutely continuous with respect to the Lebesgue measure on $I$. Under different hypothesis one can show the existence of such kind of probability measure. The literature in the subject is vast, see for instance Réinyi (1957), Lasota and Yorke (1973), Bowen (1979), Pianigiani (1980, 1981), Inoue and Ishitani (1991) and references therein, just to cite a few.

We observe that if $T$ has $s \geq 1$ full branches, then $T^{h}$ will have $s^{h}$ full branches. For $T \in \mathcal{T}$ with $s>1$ full branches, we shall denote

$K_{t}^{\uparrow}:=\left\{k:\left.T^{t}\right|_{I_{k}}\right.$ is increasing $\} \quad$ and $\quad K_{t}^{\downarrow}:=\left\{k:\left.T^{t}\right|_{I_{k}}\right.$ is decreasing $\}$.

We formally define the class of chaotic process we are interested here.

Definition 3.1. Let $T \in \mathcal{T}$ and let $\mu_{T}$ be the corresponding $T$-invariant probability measure. Let $U_{0}$ be a random variable distributed according to $\mu_{T}$ and $\varphi: I \rightarrow \mathbb{R}$ be a function in $\mathcal{L}^{1}\left(\mu_{T}\right)$. The stochastic process given by

$$
X_{t}:=\left(\varphi \circ T^{t}\right)\left(U_{0}\right), \quad \text { for all } t \in \mathbb{N},
$$

is called a $\mathcal{T}_{\varphi}$-induced process (or $\mathcal{T}_{\varphi}$ process, for short).

Figures 2(d) and 2(e) present a typical realization of the $\mathcal{T}_{\varphi}$ process associated to the Tent and Giampieri-Isola transformation, respectively. Figure 2(c) presents the graph of the transformation given by

$$
T_{a, b}(x):=\left\{\begin{array}{cll}
a+\frac{(1-a) x}{b}, & \text { if } & 0 \leq x<b, \\
\frac{a(x-b)}{1-b}, & \text { if } & b \leq x<1,
\end{array}\right.
$$

where $a, b \in(0,1)$. It can be shown that there exists an absolutely continuous $T_{a, b}$-invariant probability measure (cf. Coelho et al., 1995), but since the transformation does not have full branches, it follows that $T_{a, b} \notin \mathcal{T}$. Figure 2(f) presents a typical realization of the process given by (3.3) associated to $T_{a, b}(\cdot)$, given by $(3.4)$. Notice the contrast with the other cases. Lopes and Lopes (1998) consider large deviation properties, the spectral analysis and parameters $\alpha, \beta$ and $\sigma_{\xi}$ estimation for the signal-plus-noise process given by $Z_{t}=X_{t}+\xi_{t}=\varphi\left(T_{a, b}^{t}\left(X_{0}\right)\right)+\xi_{t}$, when $\varphi(x)=x$ and the noise is Gaussian, for different signal-to-noise ratio values. The values $\alpha$ and $\beta$ are related to the values $a$ and $b$ of the $T_{a, b}(\cdot)$ transform by the relationship $\alpha=T_{a, b}^{\prime}(x)=\frac{1-a}{b}$, if $0 \leq x<b$ and $\beta=T_{a, b}^{\prime}(x)=\frac{a}{1-b}$, if $b \leq x \leq 1$. 


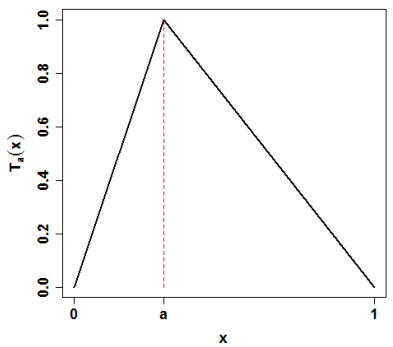

(a)

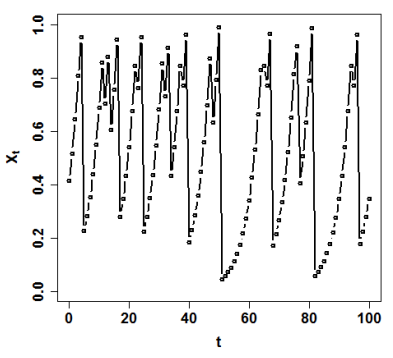

(d)

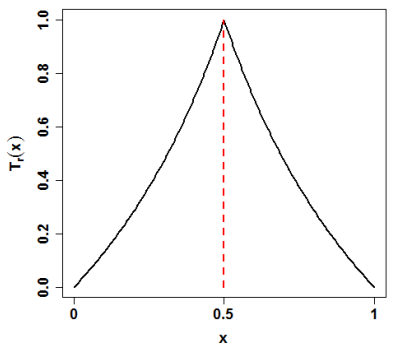

(b)

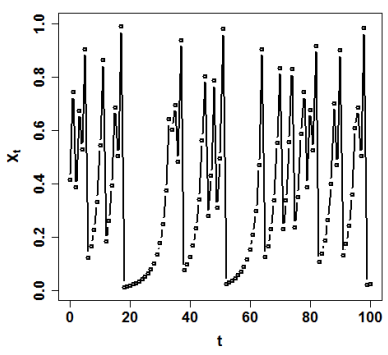

(e)

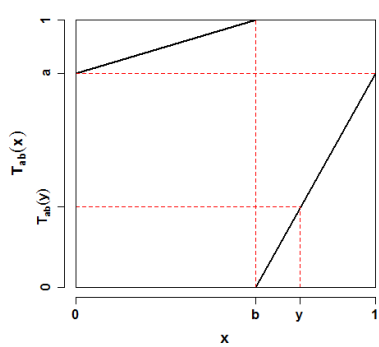

(c)

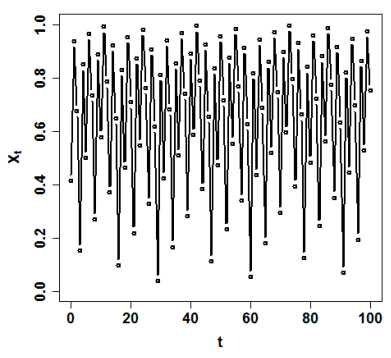

(f)

Figure 2. Typical graph of the (a) Tent transformation; (b) Giampieri-Isola transformation and (c) transformation (3.4). Typical sample from (3.3) with $T$ as (d) Tent transformation with parameter $a=0.8$; (e) Giampieri-Isola transformation with parameter $r=0.8$; (f) transformation (3.4) with parameters $a=0.8$ and $b=0.6$. In all cases $\varphi(x)=x$ and $x_{0}=\sqrt{2}(\bmod 1)$.

Given $T \in \mathcal{T}$, without further mention we shall consider that an absolutely continuous $T$-invariant probability measure has been fixed which shall be denoted by $\mu_{T}$, except otherwise explicitly said. Now let $\varphi \in$ $\mathcal{L}^{1}\left(\mu_{T}\right)$ be an almost surely increasing function and consider $\left\{X_{t}\right\}_{t \in \mathbb{N}}$ the $\mathcal{T}_{\varphi}$ process associated to $T$. For all $t \in \mathbb{N}$, let $F_{t}(\cdot)$ be the distribution function of $X_{t}$. The $T$-invariance of $\mu_{T}$, implies that, for any $t \in \mathbb{N}, t>0$, and $x \in I$,

$$
F_{t}(x):=\mathbb{P}\left(T^{t}\left(U_{0}\right) \leq x\right)=\mu_{T}\left(\left(T^{t}\right)^{-1}([0, x])\right)=\mu_{T}([0, x])=F_{0}(x) .
$$


Hence, a $\mathcal{T}_{\varphi}$ process is always strongly stationary. Also, $\mu_{T} \ll \lambda$ implies that $\mu_{T}$ is non-atomic and therefore, $F_{t}$ is continuous, increasing and its inverse is well defined.

We need to further introduce a notation for the inverse image of a singleton $y \in I$ by a transformation $T \in \mathcal{T}$. For $T \in \mathcal{T}$ with $s>1$ full branches, the restriction of $T^{h}$ to each branch $I_{k}$ defines a bijective map for which the inverse is well-defined. This inverse, denoted by $\mathcal{T}_{h, k}(y):=\left.\left(T^{h}\right)^{-1}\right|_{I_{k}}(y)$, can be then written as a vector

$$
\left(T^{t}\right)^{-1}(y)=\left(\left.\left(T^{t}\right)^{-1}\right|_{I_{1}}(y), \cdots,\left.\left(T^{t}\right)^{-1}\right|_{I_{s} t}(y)\right)=\left(\mathcal{T}_{t, 1}(y), \cdots, \mathcal{T}_{t, s^{t}}(y)\right) .
$$

\section{Copulas Related to Chaotic Processes}

In this section we shall review some of the results in the literature on copulas and chaotic process of the form (3.3). The main idea in this case is to study the copulas related to vectors coming from $\left\{X_{t}\right\}_{t \in \mathbb{N}}$. In Lopes and Pumi (2013) the authors study the chaotic process induced by the so-called Manneville-Pomeau transformation (MP transformation, for short) given by

$$
T_{s}(x)=x+x^{1+s}(\bmod 1),
$$

for $s>0$. When $s \in(0,1)$, there exists a $T_{s}$-invariant probability measure so that $T_{s} \in \mathcal{T}$ when $s \in(0,1)$. The induced chaotic process is called the Manneville-Pomeau process (MP process for short) and the copulas related to any vector of an MP process are called Manneville-Pomeau copulas (MP copulas for short). Figure 3 show the graph of the Manneville-Pomeau transformation and a typical sample path of the MP process. In Lopes and Pumi (2013) the authors derive the MP copulas in the bidimensional and multidimensional cases, when $\varphi$ is a monotone function. These copulas are shown to be singular with respect to the two-dimensional Lebesgue measure and their support, on the bidimensional case, is shown to be the lines connecting the discontinuity points of the MP transformation. We shall refrain from presenting the explicit formulas for the MP copulas here, as they are a particular case of what will be presented in the subsequent.

Lopes and Pumi (2013) also consider some computational issues, approximations and random variate generation problems related to the MP processes. In this work the authors also derive a fast procedure to estimate the underlying parameter in Manneville-Pomeau processes. In Pumi and Lopes (2013a), the authors derive the copulas related to any transformation $T \in \mathcal{T}$. Let $T \in \mathcal{T}, \mu_{T}$ be the $T$-invariant probability measure and $\left\{X_{t}\right\}_{t \in \mathbb{N}}$ be the associated $\mathcal{T}_{\varphi}$ process for $\varphi \in \mathcal{L}^{1}\left(\mu_{T}\right)$ an almost everywhere increasing function. For simplicity, let $\mathscr{F}_{h, k}: I \rightarrow\left[F_{0}\left(a_{h, k-1}\right), F_{0}\left(a_{h, k}\right)\right]$ be 


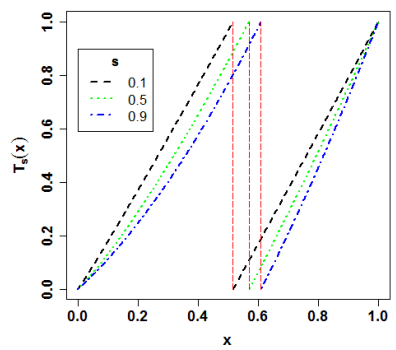

(a)

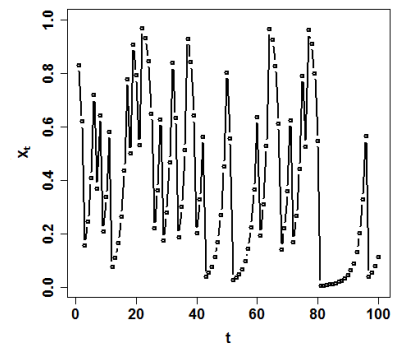

(b)

Figure 3. (a) Graph of the Manneville-Pomeau transformation for $s \in\{0.1,0.5,0.9\}$; (b) a typical sample path of an MP process.

the functions defined by

$$
\mathscr{F}_{h, k}(x):=F_{0}\left(\mathcal{T}_{h, k}\left(F_{0}^{-1}(x)\right)\right),
$$

for $h>0$ and $k \in\left\{1, \cdots, s^{h}\right\}$. For a given set $S$, we also define $\delta_{S}(u)$ as being 1 , if $u \in S$, and 0 , otherwise. If we let $\left\{a_{h, k}\right\}_{k=0}^{s^{h}}$ denote the discontinuity points of $T^{h}$, then the copula related to $\left(X_{t}, X_{t+h}\right)$ is given by

$$
\begin{aligned}
C_{X_{t}, X_{t+h}}(u, v)=\sum_{k \in n_{0}^{\uparrow}}\left[\mathscr{F}_{h, k}(v)-F_{0}\left(a_{h, k-1}\right)\right]+ \\
+\left[\min \left\{u, \mathscr{F}_{h, n_{0}}(v)\right\}-F_{0}\left(a_{h, n_{0}-1}\right)\right] \delta_{K_{h}^{\uparrow}}\left(n_{0}\right)+ \\
\quad+\sum_{k \in n_{0}^{\downarrow}}\left[F_{0}\left(a_{h, k}\right)-\mathscr{F}_{h, k}(v)\right]+\max \left\{0, u-\mathscr{F}_{h, n_{0}}(v)\right\} \delta_{K_{h}^{\downarrow}}\left(n_{0}\right),
\end{aligned}
$$

where $n_{0}:=n_{0}(u ; h)=\left\{k: u \in\left[F_{0}\left(a_{h, k-1}\right), F_{0}\left(a_{h, k}\right)\right)\right\}$, and, with $K_{h}^{\uparrow}$ and $K_{h}^{\downarrow}$ as in (3.2),

$$
n_{0}^{\uparrow}:=\left\{\begin{array}{cc}
\left\{1, \cdots, n_{0}-1\right\} \cap K_{h}^{\uparrow}, & \text { if } n_{0}>1 ; \\
\emptyset, & \text { if } n_{0}=1 ;
\end{array}\right.
$$

and

$$
n_{0}^{\downarrow}:=\left\{\begin{array}{cc}
\left\{1, \cdots, n_{0}-1\right\} \cap K_{h}^{\downarrow}, & \text { if } n_{0}>1 ; \\
\emptyset, & \text { if } n_{0}=1 .
\end{array}\right.
$$


We notice that if $T$ is taken to be the MP transformation for $s \in(0,1)$, then $n_{0}^{\downarrow}=\emptyset$ and we obtain the MP copula of $\left(X_{t}, X_{t+h}\right)$ upon the obvious substitution on (4.7) (Lopes and Pumi, 2013 applies a slightly different indexing for the sets involved). In Pumi and Lopes (2013a) it is shown that the copulas related to any bidimensional vector $\left(X_{t}, X_{t+h}\right)$ from a $\mathcal{T}_{\varphi}$ process is singular with respect to the Lebesgue measure and its support comprehend the graph of the lines joining the consecutive discontinuity points of $T^{h}$. In Figure 4 we show the three dimensional plots of the lag 2 MP copula, the level plots and 500 sample points obtained with the approximations presented.
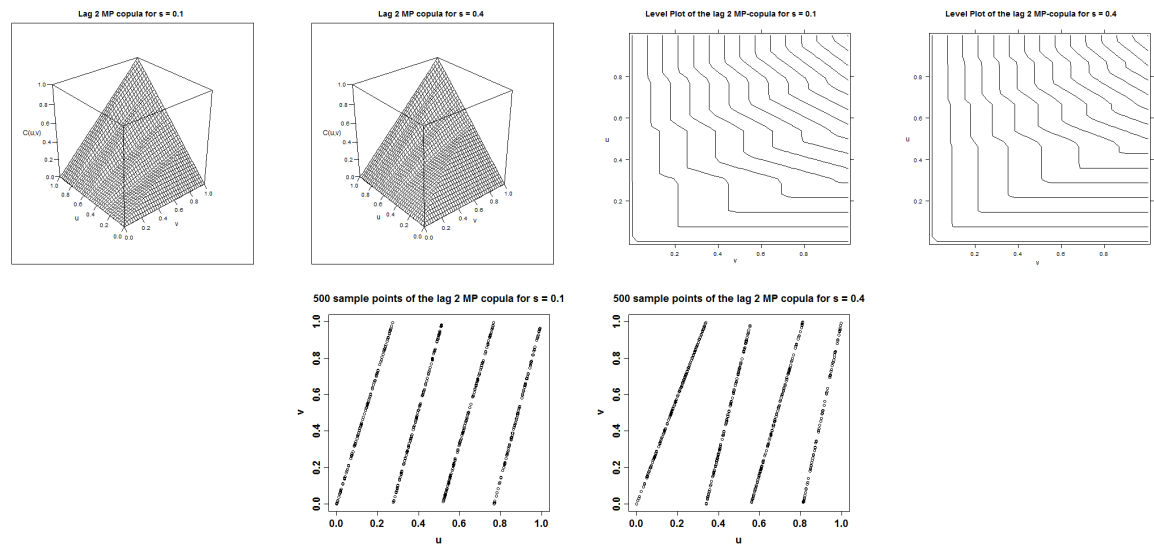

FiguRE 4. From left to right, upper panel, three dimensional plots of the lag $2 \mathrm{MP}$ copula for $s \in\{0.1,0.4\}$ and respective level sets obtained using approximations. On the bottom panel, 500 approximated sample points from a lag $2 \mathrm{MP}$ copula for $s \in\{0.1,0.4\}$

The copulas related to $n$-dimensional vectors from a $\mathcal{T}_{\varphi}$ process can also be shown to be singular with respect to the Lebesgue measure, but its support is much harder to describe. In the multivariate case, the formula of the copulas related to $\mathcal{T}_{\varphi}$ processes are involved and we shall refrain from showing them here, but they are explicitly derived in Pumi and Lopes (2013a) (and so is the multivariate MP copula in Lopes and Pumi, 2013).

4.1. The Need of Approximations. For concrete applications it is necessary to have a closed form for the copula. In order to calculate copulas of random variables coming from chaotic processes related to a $T$ map one needs an explicit form for the density of the $T$-invariant probability measure 
$\mu_{T}$. In practice, however, finding a closed form of such probability measure is usually a non-trivial task. In most cases when such a probability measure exists, it does not have a closed formula. But since the copulas depend on $\mu_{T}$ through $F_{0}$ and $F_{0}^{-1}$, non-explicit formula for $\mu_{T}$ translates as non-explicit formulas for the copulas. Another potentially complex task is determining precisely the discontinuity points of a map $T$, and if the exact location of such points cannot be analytically determined, it means that the copula cannot be explicitly determined either. So in practice there is a need to develop approximations for these (and other) elements to implement and analyze the studied copulas.

More explicitly, the elements to be known in other to determine the copulas related to bidimensional vectors coming from a $\mathcal{T}_{\varphi}$ process are the distribution function $F_{0}$ and its inverse $F_{0}^{-1}$, the inverse of $T^{h}$ in each branch $\left(\left\{\mathcal{T}_{h, k}\right\}_{k=1}^{s^{h}}\right)$ and the discontinuity points $\left\{a_{h, k}\right\}_{k=0}^{s^{h}}$ associated to $T^{h}$. In the case where $T \in \mathcal{T}$, an approximation to the $T$ invariant measure $\mu_{T}$ based on a sample of size $n$ of the respective $\mathcal{T}_{\varphi}$ process, say $x_{0}, \cdots, x_{n-1}$, at a $\mu_{T}$-continuity set $A$ is

$$
\mu_{n}\left(A ; T, x_{0}\right):=\frac{1}{n} \sum_{k=0}^{n-1} \delta_{x_{i}}(A) .
$$

An approximation $\hat{F}_{n}$ to the distribution $F_{0}$ is then obtained through the obvious relationship $\hat{F}_{n}(x):=\mu_{n}\left([0, x] ; T, x_{0}\right)$. Notice that in this context, $\hat{F}_{n}$ is just the empirical distribution calculated on the data, so that the approximation $\hat{F}_{n}^{-1}$ for $F_{0}^{-1}$ can be taken as the empirical quantile function, or an interpolation method can be used to improve the local estimation of $\hat{F}_{n}^{-1}$ based on the empirical quantile function.

In the case where $T$ is the Manneville-Pomeau transformations, Lopes and Pumi (2013) present some empirical results on the convergence of these approximations, which were found to be satisfactory and well-behaved with respect to $x_{0}$ even though, as mentioned before, the process itself is not. The authors apply a linear interpolation over the values of the empirical quantile function as approximation to $F_{0}^{-1}$.

There is no general optimal way to approximate neither the inverse image of $T^{h}$ nor its discontinuity points. In Lopes and Pumi (2013) a method based on the empirical inverse of $T^{h}$ and a linear interpolation step is used for the case of the Manneville-Pomeau transformation. A general and relatively precise way to approximate $\left\{\mathcal{T}_{h, k}\right\}_{k=1}^{s^{h}}$ and $\left\{a_{h, k}\right\}_{k=0}^{s^{h}}$ in the case $T \in \mathcal{T}$ is devised in Pumi and Lopes (2013a). The method is based on isolating the points on each branch. On each given branch, an interpolation on the point nearest to the end points of the branch and the closest one on 
the adjacent branch \pm 1 , depending whether the transformation is increasing or decreasing on the branch, is performed. However, the authors present examples where simpler and faster ways to determine the inverse image and discontinuity points of a transformation exist in some specific cases.

The approximations devised in both papers are shown to be uniformly convergent to their targets. The approximation to the copula is then obtained by plugging-in these intermediate approximations directly into the formula of the copula. Lopes and Pumi (2013) show that the approximation to the copula, in the bidimensional case, converges to the true MP copula as the sample size increases and the result holds uniformly in $I^{2}$. Pumi and Lopes (2013a) show more: in the bidimensional case, as long as the intermediate approximations applied converge uniformly to their targets, the approximation to the copula will converge uniformly in $I^{2}$ to the true copula. These results are exemplified through Monte Carlo experiments in both, Lopes and Pumi (2013) in the context of MP copulas and in Pumi and Lopes $(2013 \mathrm{a})$ in the more general case of $\mathcal{T}_{\varphi}$ processes.

The problem of random variate generation in the bidimensional case is also discussed in Lopes and Pumi (2013) and Pumi and Lopes (2013a). Given that the support of the copula of $\left(X_{t}, X_{t+h}\right)$ is simply the line connecting adjacent discontinuity points of $T^{h}$, the following general simple method can be used: take $u$ a random variate from an $U(0,1)$ distribution. This point will lie on a certain branch of $T^{h}$, say the $k$-th branch. Set $v$ as the image of $u$ by the linear function connecting the endpoints in the $k$-th branch. The desired point is then $(u, v)$.

4.2. Application to the Parameter Estimation in Chaotic Processes. Parametric estimation on a signal-plus-noise context was first considered in Lopes and Lopes (1998). Through examples that generalize the classical harmonic model, the authors present a method for obtaining the spectral distribution functions and large deviation properties of the estimated parameters of the model. Parametric estimation on MannevillePomeau processes was considered in Olbermann et al. (2007). The idea in the paper is to apply classical spectral density based estimators from the theory of long-range dependent time series in order to estimate the parameter of the Mannevile-Pomeau transformation. This idea was motivated by the fact that, on certain regions, the MP process presents slow decay of correlation (see, for details, Olbermann et al., 2007 and references therein).

In Lopes and Pumi (2013) a method for parameter estimation on MP processes inspired by the theory developed is devised. The idea is to take advantage of the simple form of the support of the MP copula to make inference on the parameter of the transformation. More specifically, given a sample $x_{0}, \cdots, x_{n-1}$ from the MP process, the idea is to form a new 
sequence $v_{i}:=\left(x_{i}, x_{i+1}\right)$, sort these vectors with respect to the branch they belong to and them locally interpolate near the discontinuity points. This can be achieved in several ways, see Lopes and Pumi (2013) for a least square and a minimum-maximum type of interpolation as well as a Monte Carlo simulation study.

In Pumi and Lopes (2013a), three methods of estimation are discussed, but two of them (curiously, the more natural and straightforward ones) are, as the authors argue, computationally too expensive for practical applications. The third method can be explained as follows. Let $T_{\boldsymbol{\theta}} \in \mathcal{T}$, with $s>1$ branches and $\boldsymbol{\theta}:=\left(\theta_{1}, \cdots, \theta_{p}\right) \in S \subseteq \mathbb{R}^{p}$, for $1 \leq p \leq s-1$. Let $\left\{a_{k}\right\}_{k=0}^{s}$ denote the discontinuity points of $T_{\boldsymbol{\theta}}$. Suppose that $\boldsymbol{\theta}$ is identifiable through the knowledge of the $\left\{a_{k}\right\}_{k=0}^{s}$ alone, that is, $\boldsymbol{\theta}:=f\left(a_{0}, \cdots, a_{s}\right)$, where $f: I^{s+1} \rightarrow S$ is a known invertible function. Let $\left\{X_{t}\right\}_{t \in \mathbb{N}}$ be the associated $\mathcal{T}_{\varphi}$ process and assume that $\varphi$ is the identity map to fix the ideas. Let $x_{1}, \cdots, x_{N}$ be a sample from $X_{t}$. Since $T_{\boldsymbol{\theta}} \in \mathcal{T}, F_{0}$ is generally smooth so that, near each of its discontinuity points $T_{\boldsymbol{\theta}}$ should behave close to a linear function. The procedure then is to identify the points that are closer to endpoints on a given branch and perform a linear interpolation between these points and their respective images by $T_{\boldsymbol{\theta}}$ (only one of then suffices).

This is a refinement of the minimum-maximum method in Lopes and Pumi (2013). The authors present a Monte Carlo simulation study on the finite sample performance of the estimator on certain chaotic process. The simulation results suggest that the procedure performs very well with small bias and small variability. Also indicates that nuisance parameters do not affect the parameter estimation.

\section{Copulas and Univariate Time Series}

In this section we present a brief survey about copula-based univariate time series. This survey does not intend to be neither a thorough nor an in-depth work, but instead, it intends to reflect the author's preferences and their research on the subject.

Copulas are fundamentally a multivariate tool for understanding and modeling joint dependence structure and marginal structure separately. Let $\left\{X_{t}\right\}_{t=0}^{\infty}$ be a weakly stationary process.

From a strictly probabilistic/measure theoretical point of view, associated to any time series, there is a sequence of distributions $\left\{F_{n}\right\}_{n=0}^{\infty}$, and to every random vector $\left(X_{t_{1}}, \cdots, X_{t_{n}}\right)$ from the process, there is an $n$ dimensional copula $C_{t_{1}, \cdots, t_{n}}$ associated to it. Describing how these copulas behave starting from an univariate time series and how one can construct a time series starting from its copulas is the subject of many studies. 
5.1. Markovian Framework. The seminal work of Darsow et al. (1992) provides a complete description of Markov processes in terms of copulas. More specifically, they studied how to translate the Chapman-Kolmogorov equations for transition probabilities into copulas framework. They also study how to specify copulas for each pair $\left(X_{r}, X_{s}\right)$ so that the resulting process is a Markov process. This technique to construct copula-based Markov process is quite different from the usual ones in that one specifies copulas and then the marginal distributions can be chosen at will. Given its importance, we outline the methodology.

Fundamentally, the idea in Darsow et al. (1992) is to determine a certain product on the space of all bidimensional copulas and then to connect this operation to the theory of Markov processes. For any pair of bidimensional copulas $C_{1}$ and $C_{2}$, it is defined a product $*$ on the space of all copulas by setting

$$
\left(C_{1} * C_{2}\right)(u, v):=\int_{I} \frac{\partial C_{1}(u, t)}{\partial t} \frac{\partial C_{2}(t, v)}{\partial t} d t .
$$

In Darsow et al. (1992), several properties of the $*$ operation are discussed. For instance, for two copulas $C_{1}, C_{2}, C_{1} * C_{2}$ is also a copula; as a binary operation in the space of copulas, $*$ is right and left distributive over linear combinations, continuous in each place (but not jointly continuous), associative and the copulas $M$ and $\Pi$ are the identity and null elements, respectively, with respect to $*$. All these properties together make the set of all copulas with the operation $*$ and the transpose operation, $C^{T}(u, v)=C(v, u)$, a symmetric Markov algebra ${ }^{1}$.

If $\left\{X_{t}\right\}_{t \in T}, T \subset \mathbb{R}$, is a continuous time process for which we denote the copula related to $\left(X_{s}, X_{t}\right)$ by $C_{s, t}$. A necessary and sufficient conditions on the copulas in order to assure that $\left\{X_{t}\right\}_{t \in T}$ is a Markov process is given in Darsow et al. (1992). In particular they show that the transition probabilities of $\left\{X_{t}\right\}_{t \in T}$ satisfy the Chapman-Kolmogorov equations if and only if $C_{s, t}=C_{s, u} * C_{u, t}$ for all $s, t, u \in T, s<u<t$. To specify a first order Markov chain in the simplest case where the marginal distributions do not vary over time (say $F$ ), based on a single bidimensional copula $C$ with density $c$, one observe that, from Sklar's theorem, the conditional density of $X_{t}$ given $X_{t-1}$ (say $f_{X_{t} \mid X_{t-1}}$ ) can be written as

$$
f_{X_{t} \mid X_{t-1}}\left(x_{t} \mid x_{t-1}\right)=c\left(F\left(x_{t-1}\right), F\left(x_{t}\right)\right) f\left(x_{t-1}\right) .
$$

\footnotetext{
${ }^{1}$ In Darsow et al. (1992), a Markov algebra is defined as a compact convex subset of a real Banach space on which a product is defined which is continuous in each place, associative, distributive over convex combinations and which possesses a null and unit elements. It is said to be symmetric if it possesses a continuous operation $T$ such that $T(T(A))=A, T(A B)=T(B) T(A)$ and it is distributive under convex combinations.
} 
More details can be found in Darsow et al. (1992) and in Section 6.4 of Nelsen (2006) and references therein. See also Lågeras (2010) which reports some non-standard behavior of copula-based Markov chains.

Joe (1997) discusses how to construct short memory time series based on conditional copulas as well as a method of constructing short memory processes based on the parameterization of the distributions on a convolution closed family. Based on the well established theory of copula-based Markov processes constructed in Darsow et al. (1992), many works studied how to characterize short memory time series based on copulas, as well as how to apply these techniques on parameter estimation. One of the first rigorous approach to this type of problem is given in Chen and Fan (2006) and later augmented in Chen et al. (2009). These works study the problem of semiparametric estimation in a copula-based univariate strictly stationary Markov processes context.

The main idea is to notice that if one apply the integral probability transform to a Markov process, the resulting process will still be a Markov process and the joint distributions are copulas. After that one can rewrite the original Markov process into a semiparametric generalized linear model depending on the copula and its parameter alone. Let $\left\{X_{t}\right\}_{t=1}^{n}$ be a sample from a stationary first order Markov process and let $F$ be the true invariant distribution. Suppose that $F$ is absolutely continuous with respect to the Lebesgue measure in $\mathbb{R}$. Let $C_{\theta}$ be the true parametric copula associated to the pair $\left(X_{t-1}, X_{t}\right)$ up to an unknown parameter $\theta$ and suppose that it is absolutely continuous with respect to the Lebesgue measure in $I^{2}$. Also suppose that $C_{\theta}$ is neither of Fréchet-Hoeffding's bounds. In this setting, if one considers a new time series by setting $U_{t}=F\left(X_{t}\right), t=$ $1, \cdots, n$, the transformed process is still a stationary Markov process, the joint distribution of $\left(U_{t-1}, U_{t}\right)$ will be $C_{\theta}(u, v)$ and the conditional density of $U_{t} \mid U_{t-1}=u_{0}$ will be $f_{U_{t} \mid U_{t-1}}\left(u \mid u_{0}\right):=c_{\theta}\left(u_{0}, u\right)$. This implies that this setting is consistent with the following semiparametric generalized linear model

$$
\varphi\left(U_{t}\right)=\psi\left(U_{t-1}\right)+\varepsilon_{t}, \quad E\left(\varepsilon_{t} \mid Y_{t-1}\right)=0,
$$

where $\varphi$ is an increasing parametric function, $\psi(\cdot)$ is given by

$$
\psi(u)=E\left(\varphi\left(U_{t}\right) \mid U_{t-1}=u\right)=\int_{0}^{1} \varphi(v) c_{\theta}(u, v) \mathrm{d} u
$$

and the conditional density of $\varepsilon_{t}$ given $U_{t-1}=u_{0}$ is given by

$$
f_{\varepsilon_{t} \mid U_{t-1}}\left(x \mid u_{0}\right)=\frac{c_{\theta}\left(u_{0}, \varphi^{-1}\left(x+\psi\left(u_{0}\right)\right)\right)}{\frac{d}{d x}\left(\varphi\left(x+\psi\left(u_{0}\right)\right)\right)} .
$$


To estimate the copula parameter, Chen and Fan (2006) apply a MLE method based on the rescaled empirical distribution while Chen et al. (2009) apply a sieve MLE methodology. The authors argue that the methodology is useful in certain financial and economic applications. A method for simulating such time series can be found in Pumi and Lopes (2010).

The study on copula-based Markov processes also includes some ergodic theoretical properties. Chen and Fan (2006) find sufficient conditions for their copula-based Markov process to be $\beta$-mixing at (least) a polynomial rate. Beare (2010) derive a condition under which a copula-based Markov process presents geometric $\beta$-mixing. This condition translates into the copula being absolutely continuous with square integrable density, a rather strong requirement that rules out, for instance, any copula presenting tail dependence or asymmetry. Beare (2012) derive sufficient conditions on the generator of an Archimedean copula so that the related Markov processes present geometric ergodicity. Chen et al. (2009) show the geometric ergodicity of copula-based Markov processes obtained from some popular copula families presenting tail dependence. Beare and Seo (2012) discuss in detail the property of time reversibility in copula-based Markov processes (in this case the copula family must be exchangeable) and introduce a test for time reversibility similar to the test of exchangeability for copulas in Nelsen (2007).

Recently the literature turned its focus to the study of higher order types of dependence. Ibragimov (2009) extends the work of Darsow et al. (1992) to higher order Markov process. In particular, as intuitively expected, it is shown that a Markov process of order $n$ is fully determined by its $n+1$-dimensional copula and its marginal distributions. Ibragimov and Lentzas (2009) discuss the slow decay of some copula-based dependence measure which motivated the authors to define a non-standard definition of "long-range dependence". They present empirical evidences that, for the Markov process based on the Clayton copula, the non-standard copulabased long-range dependence concept introduced points to the existence of "long memory" on the simulated time series. However, the theoretical results in Chen et al. (2009) show that the Markov process based on the Clayton copula actually presents short memory in the classical sense. This implies that the copula-based long-memory concepts introduced in Ibragimov and Lentzas (2009) are not compatible with the classical definition of long-range dependence.

5.2. Non-Markovian Framework. So far the works mentioned here only consider Markovian processes. The main reason for that is that outside the well-structured and well-developed theory of copula-based Markov process, it is hard to overcome the difficulties imposed by the so-called compatibility problem. In few words, the compatibility problem is an statement of the 
fact that given an arbitrary collection of $\left(\begin{array}{l}n \\ 2\end{array}\right)$ 2-dimensional copulas, there may not exist an $n$-dimensional copula whose bidimensional marginals are the given copulas. This is probably the most important open problem in the theory of copula to date.

Pumi and Lopes (2013b) present an alternative approach to deal with the covariance decay based in copulas without relying on Markovian assumptions. For a process $\left\{X_{t}\right\}_{t=0}^{\infty}$ with absolutely continuous marginal distributions $\left\{F_{t}\right\}_{t=0}^{\infty}$ and for a given parametric family of copulas $\left\{C_{\theta}\right\}_{\theta \in \Theta}$ satisfying some minor regularity conditions, the authors investigate how to translate the covariance decay in the process into a parameterization $\left\{\theta_{n}\right\}_{n=1}^{\infty}$ for the respective copula family. The only joint assumption is that the copulas related to a pair $\left(X_{r}, X_{s}\right)$ belong to a predefined parametric family, allowing the theory to cover (weakly and strongly) stationary processes as well as non-stationary ones. This condition can be actually relaxed to require that the pair $\left(X_{r}, X_{s}\right)$ has a copula $C_{\theta_{r, s}}$ belonging to a predefined family for values of $r$ and $s$ such that $|r-s|$ is large.

The main focus of Pumi and Lopes (2013b) is classical long-range dependent process, but the theory is developed in order to accommodate any type of covariance decay, including convergence to a constant, which covers the case when the variables $X_{t}$ and $X_{t+h}$ are asymptotically correlated.

The main advantage of the approach developed in Pumi and Lopes (2013b) is to be free from the compatibility problem. This is attained by focusing on pairs $\left(X_{r}, X_{s}\right)$ associating a copula $C_{\theta_{r, s}}$ and then studying the behavior of the covariance of the pair $\left(X_{r}, X_{s}\right)$ as $|r-s|$ increases. The non-standard results of Tiit (2002) on the compatibility problem provides the necessary theoretical result to show the compatibility-free nature of this approach. The copula version of Hoeffding's lemma, which connects the covariance between two random variables to its bidimensional copula and marginal distribution, is also an important ingredient on the theory.

The conditions imposed on the particular chosen parametric family of copulas, say $\left\{C_{\theta}\right\}_{\theta \in \Theta}$, are the existence of a point $\{a \in \bar{\Theta}\}$ such that $\lim _{\theta \rightarrow a} C_{\theta}(u, v)=\Pi(u, v)=u v$, the independence copula, and, as a function of its parameter, the copulas are twice differentiable in a neighborhood of $a$. As shown in Pumi and Lopes (2013b), many widely applied families of copulas satisfy such requirements. Under these assumptions the authors show that, asymptotically, the covariance decay of $\left(X_{t}, X_{t+n}\right)$ with copula $C_{\theta_{n}}$ behaves like the product of a certain function $K(n)$ (depending on the marginal distributions alone) and the behavior of the difference $\theta_{n}-a$, as $n$ increases. When the marginals of the process do not vary, $K(n)$ is a constant and, hence, the behavior of $\theta_{n}-a$ dictates the long run covariance behavior of the process. In the case where the copula family is 
multiparametric, a similar result holds, with the decay being dominated by the slowest decay among the parameter coordinates.

In Pumi and Lopes (2013b) a method for parameter estimation based on the studied theory is proposed. The method is a multi-stage procedure. Let $\left\{X_{t}\right\}_{t \in \mathbb{N}}$ be a process for which $X_{t}$ is identically distributed with common absolutely continuous distribution $F$, for all $t \in \mathbb{N}$ and let $\left\{x_{t}\right\}_{t=1}^{n}$ be a sample from it. Assume that the bidimensional copulas related to the process belong to a predetermined parametric family $\left\{C_{\theta}\right\}_{\theta \in \Theta}$ satisfying the assumptions of the previous paragraph. We also assume that the covariance of $\left\{X_{t}\right\}_{t \in \mathbb{N}}$ at lag $h$ is a known function $R(h, \gamma)$ where $\gamma$ is a parameter of interest identifiable from $R(\cdot)$. The first step in the procedure is to estimate the marginal distribution based on the given sample, say $\hat{F}$, and then form a new time series $y_{i}:=\hat{F}\left(x_{i}\right), i=1, \cdots, n$. From this new time series we construct a bidimensional process $\left\{u_{k}^{(s)}\right\}_{k=1}^{n-s}$ by $u_{i}^{(s)}:=\left(y_{i}, y_{i+s}\right), i=$ $1, \cdots, n-s$, where $s \in \mathbb{N}$ is a user-defined number called the starting lag of estimation. Next an estimate of the copula parameter related to $\left\{u_{k}^{(s)}\right\}_{k=1}^{n-s}$ is obtained, say $\hat{\theta}_{s}$. In view of the developed theory, the quantity $\hat{\theta}_{s}-a$ is regarded as an estimate of the covariance decay at lag $s$. The procedure is repeated until a sequence of estimates $\hat{\theta}_{s}, \cdots \hat{\theta}_{s+m}$ is obtained, where $m>s$ is the maximum desired lag of estimation. Finally the estimated value of $\gamma$ is set to be the value that minimizes the distance between the observed decay and the expected $R(h, \gamma)$ one through some predetermined metric.

The authors discuss large sample properties of the proposed estimator and prove its consistency under mild conditions. The authors also present a Monte Carlo study to determine the small sample properties of the proposed estimator. Simulation of stationary and non-stationary time series in this framework is also discussed and an application to real data is presented. In the next section we show an application of the method to the S\&P500.

\section{Application}

To exemplify the ideas presented on Section 5.2, we reproduce here the example given in Pumi and Lopes (2013b). The methodology is applied to the daily returns of the S\&P500 US stock market index in the period from January 01, 2000 to November 03, 2011 yielding a total sample size of $n=2,980$. Figure 5(a) to Figure 5(c) present the S\&P500 time series, the correspondent returns $\left\{r_{t}\right\}_{t=1}^{2979}$ and the absolute returns $\left\{\left|r_{t}\right|\right\}_{t=1}^{2979}$, respectively.

It is commonly found that in financial time series related to stock market indexes, the daily returns are uncorrelated while the absolute and squared returns present slow decay of covariance, usually proportional to $n^{-\beta}$ for 


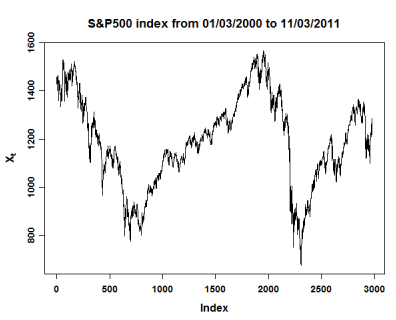

(a)

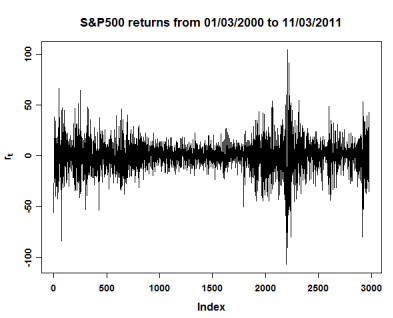

(b)

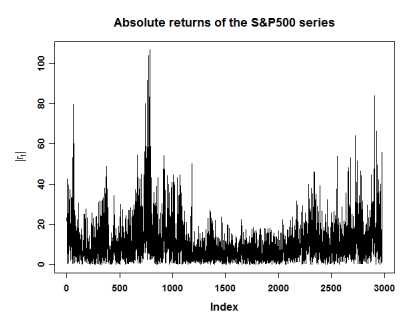

(c)

FiguRE 5. S\&P500 (a) original time series; (b) return time series; (c) absolute return time series.

$\beta \in[0.2,0.4]$ (see Cont, 2001 and references therein and also Lopes and Prass, 2013 for the intradaily case). Our goal is to apply the methodology in Pumi and Lopes (2013b) to estimate $\beta$. The study of the absolute and squared returns in financial time series are of great importance since they contain information about the (unobservable) volatility.

In order to do that we shall assume that the underlying process is strongly stationary and ergodic and that all underlying bivariate copulas belong to the same one parameter copula family. The autocorrelation and the periodogram functions of the absolute return time series of the S\&P500 index is presented in Figure 6(a). The slow decay of the sample autocorrelation function and the pronounced peak at the zero frequency in the periodogram function are commonly found in this type of data and both suggest longrange dependence on the absolute return time series. Given the behavior of the sample autocovariance function, it is often assumed that $\left\{\left|r_{t}\right|\right\}_{t=0}^{\infty}$ follows an $\operatorname{ARFIMA}(p, d, q)$ model, so that estimation of $d$ is of interest.

The first step on the methodology is to estimate the underlying marginal distribution of the data. As argue in Pumi and Lopes (2013b), an exponential distribution seems to be a good fit for the data and, by using a MLE method, an $\operatorname{Exp}(10.688)$ distribution was found to be a good fit for the data. Hence, we take $\hat{F} \sim \operatorname{Exp}(10.688)$ as the estimator for the underlying marginals and set $y_{i}=\hat{F}\left(\left|r_{t}\right|\right), t=1, \cdots, 2979$.

Next step is to identify the underlying family of bidimensional copulas related to the process. Even though in the literature, the use of the Gaussian copula on financial data sets is often contested, the authors argue that in this case it is perfectly reasonable to assume the bidimensional copulas to be Gaussian ones.

For the Gaussian copula with $\operatorname{Exp}(10.688)$ marginals, it can be show that $K_{1} \cong 260.47$ and $K_{2} \cong 40.53$. The next step is to estimate the copula 


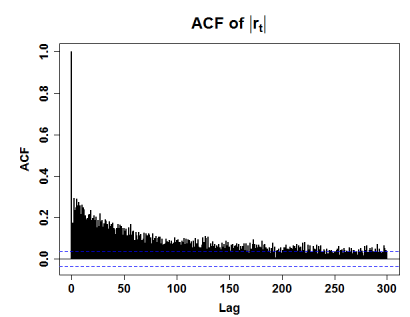

(a)

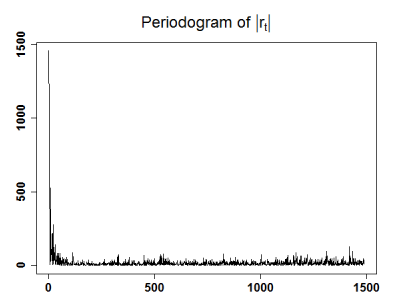

(b)

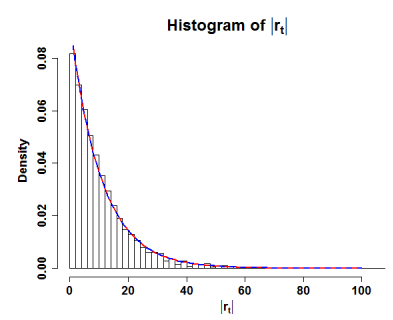

(c)

FiguRE 6. (a) Autocorrelation function; (b) periodogram function; (c) histogram of the S\&P500 absolute return time series and the fitted exponential density.

parameter $\rho$ from the pseudo samples $\left\{\left(y_{i}, y_{i+s}\right)\right\}_{i=1}^{2979-s}$. Figure 7 presents the estimated copula parameter obtained via maximum likelihood method up to lag 400. Chosen a particular lag $\ell$, let $\hat{\rho}_{1}, \cdots, \hat{\rho}_{\ell}$ denote the estimates obtained in the previous step. In Lopes and Pumi (2013b), three possible estimators, based on different functions $R(\cdot, \cdot)$ are compared. For simplicity we shall present one of the estimators. In this case we are assuming that the underlying process is an $\operatorname{ARFIMA}(0, d, 0)$ process with exponentially distributed marginals. In the literature, the correlation decay on the squared returns is observed to be proportional to $n^{-\beta}$ for $\beta \in[0.2,0.4]$ (see Cont, $2001)$ and the relationship to the parameter $d$ of an $\operatorname{ARFIMA}(0, d, 0)$ model is $\beta=1-2 d$. The estimator is defined through the following optimization procedure:

$$
\hat{d}:=\underset{d \in(-0.5,0.5)}{\operatorname{argmin}}\left\{\frac{1}{m+1} \sum_{h=s}^{s+m}\left|\hat{\rho}_{h}-\Gamma(d)^{-1} h^{2 d-1}\right|\right\}, \quad \hat{\beta}:=1-2 \hat{d} .
$$

For the data, $\hat{d}=0.3370$ with $95 \%$ confidence interval $(0.3320,0.3432)$ which gives $\hat{\beta}=0.3260$ with $95 \%$ confidence interval $(0.3136,0.3360)$, all coherent with the findings reported on literature (see Cont, 2001 and references therein). These findings are also consistent with non-linear modeling of the S\&P500 data as reported, for instance, in Prass and Lopes (2013) (see also references therein).

\section{Conclusions}

In this work we present a brief summary on the modern and classical results on the role played by copulas in the theory of chaotic processes and univariate time series. 


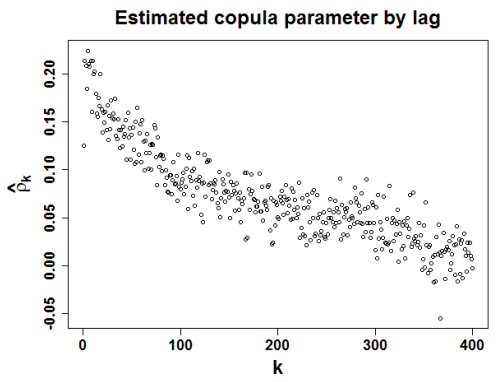

FiguRE 7. Copula parameter estimated values.

In Section 1 and 2 the problem is introduced and some results on copulas are briefly reviewed. In Section 3 we briefly review some facts on the theory of chaotic processes necessary to the work. We formally define chaotic processes, discuss some of its properties and introduce the class of transformations considered in the reviewed literature. We proceed in Section 4 to review the connection between copulas and chaotic process and some of its properties.

The copulas related to the class of chaotic processes considered here usually do not present closed formulas so that the study and application of such copulas rely on approximations. Some ideas on the approximations presented in the literature as well as applications to parameter estimation in chaotic process and random variate generation are also discussed.

In Section 5 we briefly review the connections between copulas and univariate time series through the copula lens. The section is divided in Markovian and non-Markovian theory. In the Markovian case, we review some of the ideas in the seminal paper by Darsow et al. (1992) on the theory of copula-based Markov processes as well as some applications to the parameter estimation in copula-based (short memory) time series. We also briefly review some results on the ergodic properties of copula-based Markov processes. Some extensions are also discussed.

In the non-Markovian case we shortly discuss the compatibility-free approach to general dependence in Pumi and Lopes (2013b), focusing on the ideas behind the theory developed there and its applications. We also provide an application of the methodology to real time series.

Acknowledgements. S.R.C. Lopes research was partially supported by CNPq-Brazil, by CAPES-Brazil, by Pronex Probabilidade e Processos Estocásticos - E-26/170.008/2008 -APQ1 and also by INCT em Matemática. 


\section{References}

[1] Bowen, R. (1979). "Invariant Measures for Markov Maps of the Interval". Communications in Mathematical Physics, 69, 1-14.

[2] Beare, B.K. (2010). "Copulas and temporal dependence". Econometrica, 78(1), 395-410.

[3] Beare, B.K. (2012). "Archimedean copulas and temporal dependence". Econometric Theory, 28(6), 1165-1185.

[4] Beare, B.K. and Seo, J. (2012). "Time Irreversible Copula-Based Markov Models". University of California at San Diego, Economics Working Paper Series.

[5] Chazottes, J.-R., Collet, P. and Schmitt, B. (2005). "Statistical Consequences of the Devroye Inequality for Processes. Applications to a Class of Non-Uniformly Hyperbolic Dynamical Systems". Nonlinearity, 18(5), 2341-2364.

[6] Chen, X. and Fan, Y. (2006). "Estimation of Copula-Based Semiparametric Time Series Models". Journal of Econometrics, 130, 307-335.

[7] Chen, X., Wu, W.B. and Yi, Y. (2009). "Efficient Estimation of Copula-Based Semiparametric Markov Models". Annals of Statistics, 37, 4214-4253.

[8] Cherubini, U., Luciano, E. and Vecchiato, W. (2004). Copula Methods in Finance. Chichester: Wiley.

[9] Coelho, Z., Lopes, A.O. and da Rocha, L.F. (1995). "Absolutely Continuous Invariant Measures for a Class of Affine Interval Exchange Maps". Proceedings of the American Mathematical Society, 123(11), 3533-3542.

[10] Darsow, W.F., Nguyen, B. and Olsen, E.T. (1992). "Copulas and Markov processes". Illinois Journal of Mathematics, 36(4), 600-642.

[11] Giampieri, M. and Isola, S. (2005). "A One-Parameter Family of Analytic Markov Maps with an Intermittency Transition". Discrete and Continuous Dynamical Systems, 12(1), 115-136.

[12] Ibragimov, R. (2009). "Copula-based characterizations for higher order Markov processes". Econometric Theory, 25, 819-846.

[13] Ibragimov, R. and Lentzas, G. (2009). "Copulas and long memory". Harvard Institute of Economic Research. Discussion Paper No. 2160.

[14] Inoue, T. and Ishitani, H. (1991). "Asymptotic Periodicity of Densities and Ergodic Properties for Nonsingular Systems". Hiroshima Mathematical Journal, $\mathbf{2 1}(3), 597-620$.

[15] Joe, H. (1997). Multivariate Models and Dependence Concepts. New York: Chapman \& Hall.

[16] Kolesárová, A., Mesiar, R. and Sempi, C. (2008). "Measure-preserving Transformations, Copulæ and Compatibility". Mediterranean Journal of Mathematics, 5(3), 325-339.

[17] Lagerås, A.N (2010). "Copulas for Markovian Dependence". Bernoulli, 16(2), 331-342.

[18] Lasota, A. and Mackey, M.C. (1994). Chaos, Fractals, and Noise. Stochastic Aspects of Dynamics. New York: Springer-Verlag. 2nd. Edition.

[19] Lasota, A. and Yorke, J.A. (1973). "On the Existence of Invariant Measures for Piecewise Monotonic Transformations". Transactions of the American Mathematical Society, 186, 481-488.

[20] Lee, T-H. and Long, X. (2009). "Copula-based multivariate GARCH model with uncorrelated dependent errors". Journal of Econometrics, 150(2), 207-218.

[21] Lopes, A. and Lopes, S.R.C. (1998). "Parametric Estimation and Spectral Analysis of Piecewise Linear Maps of the Interval". Advances in Applied Probability, 30, 757-776. 
[22] Lopes, S.R.C. and Prass, T.S. (2013). "Seasonal FIEGARCH Processes". Computational Statistics and Data Analysis, 68, 262-295.

[23] Lopes, S.R.C. and Pumi, G. (2013). "Copulas Related to Manneville-Pomeau Processes". Brazilian Journal of Probability and Statistics. 27(3), 322-356.

[24] Lopes, S.R.C., Pumi, G. and Zaniol, K. (2011). "Mallows distance in VARFIMA $(0, \boldsymbol{d}, 0)$ processes". Communications in Statististics: Simulation and Compututation, 42(1), 24-51.

[25] Mari, D.D. and Kotz, S. (2001). Correlation and Dependence. London: Imperial College Press.

[26] McNeil, A.J., Frey, R. and Embrechts, P. (2005). Quantitative Risk Management: Concepts, Techniques and Tools. Princeton: Princeton University.

[27] Nelsen, R.B. (2006). An Introduction to Copulas. New York: Springer-Verlag. 2nd. Edition.

[28] Nelsen, R.B. (2007). "Extremes of nonexchangeability". Statistical Papers, 48, 329-336.

[29] Olbermann, B.P, Lopes, S.R.C and Lopes, A.O. (2007). "Parameter Estimation in Manneville-Pomeau Processes". Unpublished manuscript. Source: arXiv:0707.1600.

[30] Palynchuk, B.A. and Guo, Y. (2011). "A probabilistic description of rain storms incorporating peak intensities". Journal of Hydrology, 409(1-2), 71-80.

[31] Pianigiani, G. (1980). "First Return Map and Invariant Measures". Israel Journal of Mathematics, 35, 32-48.

[32] Pianigiani, G. (1981). "Existence of Invariant Measures for Piecewise Continuous Transformations". Annales Polonici Mathematici, 40, 39-45.

[33] Pollicott, M. and Yuri, M. (1998). Dynamical Systems and Ergodic Theory. Cambridge: Cambridge University Press.

[34] Pumi, G. and Lopes, S.R.C. (2010). "Simulation of Univariate Time Series Using Copulas". In: Atas do $19^{\circ}$ Simpósio Nacional de Probabilidade e Estatística.

[35] Pumi, G. and Lopes, S.R.C. (2013a). "Copulas Related to Piecewise Monotone Functions of the Interval and Associated Processes". Under review.

[36] Pumi, G. and Lopes, S.R.C. (2013b). "Parameterization of Copulas and Covariance Decay of Stochastic Processes with Applications". Under review. Preprint arXiv:1204.3339

[37] Rényi, A. (1957). "Representations for Real Numbers and their Ergodic Properties". Acta Mathematica Academiae Scientiarum Hungaricae, 8, 477-493.

[38] Rüschendorf, L. (2009). "On the Distributional Transform, Sklar's Theorem, and the Empirical Copula Process". Journal of Statistical Planning and Inference, 139(11), 3921-3927.

[39] Schmidl, D.; Czado, C.; Hug, S. and Thesis, F.J (2013). "A Vine-copula Based Adaptive MCMC Sampler for Efficient Inference of Dynamical Systems". Bayesian Analysis, 8(1), 1-22.

[40] Schweizer, B (1991). "Thirty years of copulas". In Advances in probability distributions with given marginals (eds. Dall'Aglio, G.; Kotz, S.; and Salinetti, G.), 13-50, Kluwer Acad. Publ., Dordrecht.

[41] Schweizer, B. and Sklar, A. (2005). Probabilistic Metric Spaces. Mineola: Dover Publications.

[42] Sklar, A. (1959). "Fonction de Repartition à $n$ Dimensions et Leurs Marges". Publications of the Institute of Statistics of University of Paris, 8, 229-231.

[43] Tiit, E. (2002). "Existence of distributions with given marginals". In Distributions With Given Marginals and Statistical Modeling (eds. Cuadras, C.M. , Fortiana, J. and Rodriguez-Lallena, J.A.), 229-242. Kluwer, Dordrecht. 
[44] Wang, D., Rachev, S.T. and Fabozzi, F.J. (2009). "Pricing of Credit Default Index Swap Tranches with One-Factor Heavy-Tailed Copula Models". Journal of Empirical Finance, 16(2), 201-215.

[45] Zebrowsky, J.J. (2001). "Intermittency in Human Heart Rate Variability". Acta Physica Polonica B, 32, 1531-1540. 